\title{
Ananlysis of Element-wise Response under Uniform Three-dimensional Deformation Condition
}

\author{
Binbin $\mathrm{Xu}^{1, \text { a }}$ and Wei $\mathrm{Si}^{2, \mathrm{~b}}$ \\ ${ }^{1}$ Tianjin Port Engineering Institution Ltd. of CCCC, Tianjin, China; Key Lab. of Geotechnical \\ Engineering of Tianjin, Tianjin, China; Key Lab. of Geotechnical Engineering, Ministry of \\ Communication, Tianjin, China \\ 2 Tianjin Port Engineering Institution Ltd. of CCCC, Tianjin, China \\ axubinbin@tpei.com.cn, bsiwei@tpei.com.cn
}

Keywords: Element-wise response, Three-dimensional, Uniform deformation, Numerical stability Abstract. In order to get the mechanical response of soils, the element-wise experiments are always carried out assuming a uniform deformation field. In this paper, the attempt to obtain uniform deformation field under three dimensional cubic and cylinder conditions is made theoretically. It is found that the pore water pressure inside the specimen is determined by the horizontal accelerations. While in the cubic condition, the horizontal acceleration is not unique, and it is impossible to realize the uniform deformation field. However, for the cylinder condition, the uniform field is feasible.

\section{Introduction}

No matter in the indoor experiment or in the numerical analysis, a uniform deformation field is always preferred compared with other deformation, which is the basement to verify the validation of one constitutive model or to grasp the critical mechanical properties of certain soil. Previously, many researchers have carried out such researches based on the one-dimensional or two-dimensional uniform deformation field $[1,2,3]$. Due to the deformation of the specimen, there would be strain localization or localized deformation when the strain becomes large and it is nearly impossible to reproduce the uniform deformation field.

In this paper, a three-dimensional uniform deformation is attempted by considering the theoretical equations in the deformation field. During the deformation process, an initial velocity field is applied to each node of the specimen and accordingly an initial pore water pressure field is also applied to each element of the specimen artificially to eliminate the inertia effect and a uniform deformation field can be finally obtained based on the triaxial three-dimensional condition. The numerical stability during the calculation is discussed in addition.

\section{Three Dimensional Cubic Condition}

In this section, the general case is considered for an undrained compression test on a three dimensional cubic condition using a soil-water coupled analysis. Consider a vertical extension or compression test with constant strain rate on a perfectly cubic specimen under undrained conditions between rigid and frictionless pedestals at both the top and bottom ends. The Descartes coordinate system is applied into the inertia system, with the reference coordinate in the horizontal and vertical directions, $X_{1}, X_{2}$ and $X_{3}$, respectively, the current coordinate in the horizontal and vertical directions, $x_{1}, x_{2}$ and $x_{3}$, respectively, as shown in Fig. 1. Here, $\delta$ and $v(t)$ are the deformation rate at the top surface and surrounding surface respectively. The uniform deformation field of the soil skeleton can be represented by Eq. 1 . 


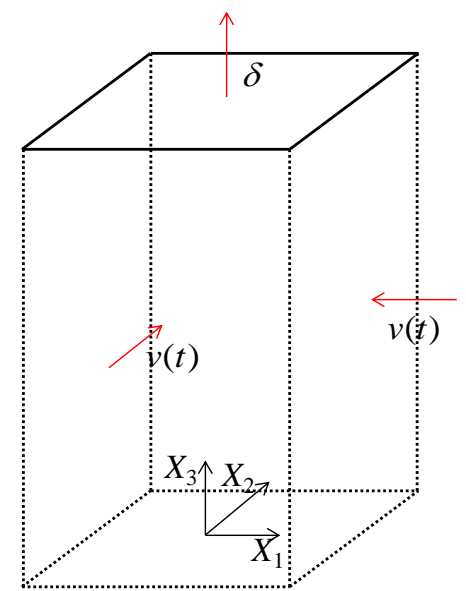

Figure 1 Sketch of specimen under three-dimensional cubic condition

$$
\left\{\begin{array}{l}
x_{1}=\frac{1}{\sqrt{1+\delta t}} X_{1} \\
x_{2}=\frac{1}{\sqrt{1+\delta t}} X_{2} \\
x_{3}=(1+\delta t) X_{3}
\end{array}\right.
$$

where $0 \leq t<+\infty$ when $\delta>0$ and $0 \leq t<\frac{-1}{\delta}$ when $\delta \leq 0$, and $x_{1} x_{2} x_{3}=X_{1} X_{2} X_{3}$ expresses that the specimen deforms element-wise in an undrained (constant volume or no volumetric change) condition.

Correspondingly, the velocity and acceleration in each direction are determined by:

$$
\left\{\begin{array} { c } 
{ \dot { x } _ { 1 } = - \frac { 1 } { 2 } ( 1 + \delta t ) ^ { - \frac { 3 } { 2 } } \delta X _ { 1 } } \\
{ \dot { x } _ { 2 } = - \frac { 1 } { 2 } ( 1 + \delta t ) ^ { - \frac { 3 } { 2 } } \delta X _ { 2 } } \\
{ \dot { x } _ { 3 } = \delta X _ { 3 } }
\end{array} \quad \left\{\begin{array}{c}
\ddot{x}_{1}=\frac{3}{4}(1+\delta t)^{-\frac{5}{2}} \delta^{2} X_{1} \\
\ddot{x}_{2}=\frac{3}{4}(1+\delta t)^{-\frac{5}{2}} \delta^{2} X_{2} . \\
\ddot{x}_{3}=0
\end{array}\right.\right.
$$

As can be seen, when the vertical velocity at the top surface is constant, the vertical acceleration at the top surface is zero and there is no inertia effect in the vertical direction, therefore the focus should be put on the horizontal directions. According to the equilibrium equations in two horizontal directions,

$$
\left\{\begin{array}{l}
\rho \ddot{x}_{1}=\frac{\partial T_{1}}{\partial x_{1}}=\frac{\partial T_{1}^{\prime}}{\partial x_{1}}-\frac{\partial u}{\partial x_{1}} \\
\rho \ddot{x}_{2}=\frac{\partial T_{2}}{\partial x_{2}}=\frac{\partial T_{2}^{\prime}}{\partial x_{2}}-\frac{\partial u}{\partial x_{2}}
\end{array} .\right.
$$

and the uniform deformation assumption, then the relationship between the pore water pressure and the acceleration in the horizontal directions can be obtained as follows:

$$
\left\{\begin{array}{l}
\frac{\partial u}{\partial x_{1}}=-\rho \ddot{x}_{1} \\
\frac{\partial u}{\partial x_{2}}=-\rho \ddot{x}_{2}
\end{array} .\right.
$$


If we integrate the Eq. 4 and the following equation can be got:

$$
\left\{\begin{array}{l}
u=-\rho \int_{x_{1}} \ddot{x}_{1} d x_{1} \\
u=-\rho \int_{x_{2}} \ddot{x}_{2} d x_{2}
\end{array} .\right.
$$

It can be seen that in one element, the pore pressure is determined by two different equations. If and only if $\ddot{x}_{1}=\ddot{x}_{2}$, the pore pressure is unique, which means that there will be two pore pressures inside one element if $\ddot{x}_{1} \neq \ddot{x}_{2}$. Therefore, it is impossible to realize the uniform deformation in the three dimensional cubic condition.

\section{Three Dimensional Triaxial Condition}

In this section, the case for an undrained compression test on a three-dimensional cylinder condition is carried out. Consider a vertical extension/compression test with constant strain rate on a perfectly cylinder specimen under undrained conditions. The cylindrical-coordinate system is applied into the inertia system, with the reference coordinate in the radial, circumferential and vertical directions, $R$, $\Theta$ and $Z$, respectively, the current coordinate in the horizontal and vertical directions, $r, \theta$ and $z$, respectively, as shown in Fig. 2. The uniform deformation field of the soil skeleton can be represented by Eq. 6 .

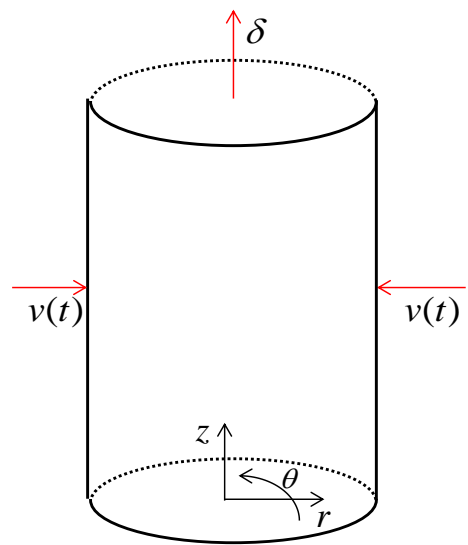

Figure 2 Sketch of uniform deformation field for cylinder condition

$$
\left\{\begin{array}{l}
r=\frac{1}{\sqrt{1+\delta t}} R \\
z=(1+\delta t) Z
\end{array} .\right.
$$

where $0 \leq t<+\infty$ when $\delta>0$ and $0 \leq t<\frac{-1}{\delta}$ when $\delta \leq 0$. The corresponding velocity and acceleration are:

$$
\left\{\begin{array}{c}
\dot{r}=-\frac{1}{2}(1+\delta t)^{-\frac{3}{2}} \delta R \\
\dot{z}=\delta Z
\end{array},\left\{\begin{array}{c}
\ddot{r}=\frac{3}{4}(1+\delta t)^{-\frac{5}{2}} \delta^{2} R \\
\ddot{z}=0
\end{array}\right.\right.
$$

Again we can see that there is no acceleration in the vertical direction. According to the equilibrium equation in the radial direction,

$$
\rho \ddot{r}=\frac{\partial T_{r r}}{\partial r}+\frac{T_{r r}-T_{\theta \theta}}{r} .
$$


and the uniform deformation assumption, that is $\frac{\partial T_{r r}{ }^{\prime}}{\partial r}=0$ and $T_{r r}-T_{\theta \theta}=0$, then we can get the relationship between the gradient of pore pressure and acceleration in the radial direction:

$$
\frac{\partial u}{\partial r}=-\rho \ddot{r}
$$

Substitute Eq. 7 into the above equation, the pore pressure expressed by Eulerian and Lagrangian descriptions is:

$$
u(r, t)=\frac{-3 \rho \delta^{2}}{8(1+\delta t)^{2}} r^{2}+A(t), u(R, t)=\frac{-3 \rho \delta^{2}}{8(1+\delta t)^{3}} R^{2}+A(t) .
$$

where $A(t)$ represents a function of time $t$, which is determined by using effective stress principle and stress boundary condition. According to the boundary condition, then $A(t)$ is:

$$
A(t)=c+T^{\prime}(0)+\frac{3}{8} \rho \delta^{2} R_{0}^{2}=\frac{3}{8} \rho \delta^{2} R_{0}^{2}
$$

Therefore, the pore pressure due to the acceleration along the radial direction is:

$$
u(r, 0)=-\frac{3}{8} \rho \delta^{2}\left(r^{2}-R_{0}^{2}\right)
$$

and it is a curved surface with a symmetrical axis $r=R=0$.

Compared with the three-dimensional cubic condition, it is more suitable to use three dimensional cylinder specimens to reproduce the element-wise response and actually in the practice the triaxial tests which are quite similar to the three dimensional cylinder conditions are usually used to determine the basic mechanical properties of soils. In addition, in order to avoid the restriction of the frictional pedestal at both ends as much as possible, the lubricating oil is also used to relief the influence of friction.

\section{Summary}

In this paper, the element-wise response under three dimensional conditions are discussed in details and the cubic and cylinder conditions are presented by assuming a uniform deformation field under undrained compression or expansion tests. The conclusions are as follows:

(1) When a constant vertical rate is applied at the top surface of the cubic specimen, there is no vertical acceleration and the horizontal acceleration occurs. But because the pore water pressure is determined by the accelerations at two horizontal directions, it is impossible to obtain uniform deformation under cubic condition.

(2) For the cylinder condition, because the accelerations at all the radius direction are same in every horizontal direction, the pore water pressure is uniquely determined and the uniform deformation field is possible. Therefore, the triaxial tests are preferred for the element-wise response.

\section{References}

[1] C. Yatomi, A. Yashima, A. Iizuka and I. Sano: Soils and Foundations Vol. 29 (1989) p. 41.

[2] R. Hill and J. W. Hutchinson: Mech. Phys. Solids Vol. 23 (1975) p. 239.

[3] R. G. Wan, D.H. Chan and N. R. Morgenstern: Finite Elements in Analysis and Design 7 (1990) p. 129. 